\title{
Du français, dlalang et des poèmes incorrects : langage et poétique chez Katalin Molnár
}

\author{
Lucie Bourassa \\ Université de Montréal
}

Les écrits de Katalin Molnár demandent à être non seulement lus, mais également vus, proférés et entendus, autrement dit, appréhendés dans leur matérialité : cela vaut tout autant pour les entours que pour l'œuvre proprement dite.

\section{Unproblèm téorik surlelangaj}

C'est pourquoi je propose ici un premier extrait de cette création verbale aux apparences multiples. Le passage est puisé 
dans le texte d'ouverture de la très éphémère Poézi prolétèr, une revue que Molnár fonda en 1997 avec Christophe Tarkos et Pascal Doury :

parske sètrevu, sépaünrevu. élè lié ôlangaj, si tu veû. ya unproblèm téorik, ya unproblèm téorik surlelangaj. oui, surlelangaj.

é, échpanske si onfé juste sa, si onsekonsantr ladsu, onsksantr just ladsu, sa peû avoir dlavaleur. sa peû ètr intérèsan. échtrouv, si tu veû, danlarnak jénéralizé, ilgna plurien ki peû ètr klèr, klèrman di ladsu parske lèzanjeû, léproblématik, èlsedéplas vèrdéchjôz insignifiant. donk, ilfô, ilfô, ilfô reprandr trankilman letruk édir dé, redir déchôz toutsinpl, ki onbezoin de, de réafirmé. (Molnár, 1997, p. 15)

Après avoir déchiffré ce passage, on aura peut-être l'impression que cette revue est un rejeton des périodiques littéraires et théoriques des années 1960 et 1970, Tel Quel, Change ou TXT par exemple, mais dans une version défigurée, ou plutôt, actualisée, contaminée par la hâtive graphie des SMS ou des chats, ainsi que par l'oralité des rappeurs, slameurs et autres performeurs. Dans la table des matières, le texte d'introduction est attribué à Katalin Molnár seule. À la première page, on voit cependant les mots "diskusion antr», suivis de deux photos séparées par la "préposition » "é ». Ces photos présentent les deux interlocuteurs (Molnár et Tarkos) dans des tenues et mises en scène assez loufoques, propres à rappeler Dada ou Fluxus : l'une des images montre une femme arborant un seau à eau sur la tête et tenant un papier et un micro; sur la deuxième apparaît un homme vêtu d'un t-shirt et d'une veste, les jambes nues entre ses larges boxers et ses chaussettes. Dans leur "diskusion », les deux interlocuteurs parlent des raisons d'être de leur revue, de ce qu'ils y publieront et de la manière dont ils s'y prendront pour la fabriquer et la diffuser. 
En qualifiant leur publication de « revue semestrielle de poésie contemporaine et de recherche expérimentale sur la langue française ${ }^{1}$ », Doury, Molnár et Tarkos se rattachent à une tradition qui remonte à la deuxième moitié du XIX siècle. Dans un article intitulé «Poésie de la linguistique : la tentation du dictionnaire ", Hugues Laroche retrace les liens entre les recherches de la linguistique naissante, en particulier au plan du lexique, et certains textes de poètes comme Hugo, Gourmont, Adam, Schwob ou Mallarmé. À partir de ce moment, se déploie en France une tradition de poètes qui se sont absorbés dans des réflexions, méditations, rêveries sur le langage, tradition qui d'ailleurs ne doit pas tout à l'influence de la linguistique.

Avec ses doléances sur l'absence de motivation des langues et sa critique de l'usage instrumental du langage, Mallarmé assigne une nouvelle fonction à la poésie, celle de « rémunérer » le « défaut des langues » (p. 208). De nombreux poètes français l'ont suivi, accordant au langage une importance centrale, à la fois dans des réflexions explicites (qui proposent des représentations du langage souvent fort critiques) et dans la pratique (l'invention de nouveaux modes de signifier). Parmi les doléances les plus récurrentes dans leurs réflexions linguistiques figurent celles de l'arbitraire des langues et du caractère aliénant du langage partagé, vu par exemple comme un «tas de vieux chiffons pas à prendre avec des pincettes » (Ponge, 2003, p. 196), une «langue de muesli», «molle, caoutchoutée, médiocre, laide» (Roubaud, 1995 p. 35-37), la «langue de tous [qui] n'est celle de personne » (Prigent, 19921993 p. 32). Les solutions poétiques proposées (dans la théorie comme dans l'écriture) connaissent autant de variations que les

\footnotetext{
${ }^{1}$ C'est le sous-titre de la revue, tel qu'il apparaît à l'endos de la couverture.
} 
critiques du langage. Elles consistent souvent en une tentative de remotiver la langue ou encore en une résistance à sa dimension aliénante, motivation et résistance pouvant s'associer, par exemple chez Artaud, qui espérait trouver dans la glossolalie une langue du corps contre la "parole soufflée ", ou chez Prigent, qui cherche un idiome apte à «verbaliser l'intime» contre «l'homogénéité verbalisée de la communauté » (p. 32).

C'est ce lien entre théorie du langage et poétique que j'analyserai ici dans les «entours» de l'œuvre de Katalin Molnár, écrivaine née en Hongrie en 1951 et émigrée en France en 1979. Molnár commença à publier dans son pays d'adoption, des poèmes en hongrois d'abord, puis des textes en français ensuite. Dans cette dernière langue, elle a fait paraître quatre livres: deux ouvrages poétiques, poèmesIncorrects et mawvaisChants chantsTranscrits et Quant à je (kantaje), un essai, Konférans pour léz ilétrés, et un roman, Lamour-Dieu. Elle a aussi publié des textes dans des collectifs, notamment un essai sur la langue et la littérature intitulé « Dlalang », dans la Revue de littérature générale en 1996, et deux textes de réflexion dans Poézi prolétèr 1, «Diskusion» et «Du français». Parmi ces travaux, la part occupée par les entours - essais autonomes et paratextes - est très importante. Le cas le plus spectaculaire est celui du premier livre, poèmesIncorrects et mauvaisChants chantsTranscrits ${ }^{2}$, où presque toutes les pages de gauche sont occupées par du paratexte, surtout des notes, parfois un document ou une photographie. Une bonne partie des écrits de Molnár traite par ailleurs du langage et, dans une moindre

\footnotetext{
2 J'appelle ici «premier livre » le premier titre paru en français, puisque je n'étudie pas les poèmes parus en hongrois.
} 
mesure, de littérature ou de poésie. Cependant, les catégories " entours » et "discours théorique » ne se recoupent pas : les notes de poèmesIncorrects et de Quant à je (kantaje) narrent souvent des circonstances d'écriture des textes; le corps du livre Quant à je contient, par contre, beaucoup de passages métalinguistiques et métalittéraires, à commencer par une suite intitulée "Quant à je », qui est une forme d'art poétique, mais défini par la négative. La séparation entre l'œuvre proprement dite et ses marges n'est donc pas nette. Je me concentrerai malgré tout ici sur les documents qui sont clairement des entours, soit les essais et les paratextes (quatrièmes de couverture, préfaces, notes), auxquels viendra s'ajouter une interview réalisée pour la revue électronique Libr-critique à l'occasion de sa participation au Festival Expoésie de Périgueux en 20073 .

\section{Ô kruèl souvenir de ma gloire passé !}

Par rapport à celle des autres poètes français qui ont proposé des méditations, rêveries ou réflexions sur le langage, la pensée de Molnár a ceci de particulier qu'elle prend explicitement sa source dans des expériences concrètes de sa vie personnelle. Dès la première note des poèmesIncorrects..., l'écrivaine raconte - usant déjà de manière approximative d'une écriture phonétique dont elle fixera un peu plus tard les règles ${ }^{4}$ - une partie de son histoire avec les langues hongroise et française, cela pour donner un arrière-plan au premier syntagme de son titre :

3 On peut voir la vidéo de cette entrevue à l'adresse : http://www.t-pasnet.com/libr-critique/?p=734.

${ }^{4}$ Dans « Du français » et Konférans pour lé zilétrés. 
Ma mèr, né d'unn mèr ôtrichyènn, a été séparé d'èl a l'aj de deu ou troa zan, é n'a plu parlé du tou pandan pluzyoer zané. Par la suit, èl a toujour parlé oen ongroa ènsèrtèn é mal aksantué, oen peu kom unn nétranjèr. Ankor ôjourd'ui, très souvan èl ne sè pa koman finir sé fraz. Èl se lans dan zunn fraz é s'arèt ô milyeu é réfléchi sur lé fasson d'aboutir. Pour surmonté sèt difikulté èl se sèr régulyèrman dé tournur don ton napran l'uzaj ô parti komunist. Mon pèr pratikè ôssi sé tournur. Tou deu, manbr du parti é kadr fidèl du réjim an plas alor, lizè bôkou de litratur idéolojik, suivè dé kour idéolojik, étsétéra. Mon pèr, issu d'unn famiy ouvryèr (kom ma mèr d'ayoer) fezè ossi dé fôt de lang ki étè konsidéré kom inadmissibl par lé milyeu kultivé. De sèt famiy é de se réjim j'é donk érité d'unn trè grand ènsèrtitud kan ta la bonn fasson de parler é d'ékirir an ongroa é sessi malgré mé long zétud lèngouistik é litérèr an lang ongroaz. (1995, p. 2, note 1)

Elle ajoute ensuite qu'elle a dû apprendre le français à l'âge de quatorze ans, qu'elle est devenue professeure de littérature et de linguistique française, mais une professeure qui n'était jamais allée en France et qui, lorsqu'elle s'y installa, n'avait pu corriger toutes ses fautes, solidement ancrées: "J'é par konsékan ôssi unn trè grand ènsèrtitud kan ta la bonn fasson de parlé é d'ékrir an fransè », conclut-elle. Dans Konférans pour lé zilétrés, on apprend que le français n'a jamais été un choix pour elle: adolescente, elle voulait étudier l'anglais à cause des Beatles, mais le directeur de son lycée a décidé qu'elle ferait plutôt du français, parce que les rockeurs de Liverpool avaient donné à trop d'élèves l'envie de parler leur langue. L'apprentissage imposé du français fut, pour le moins, pénible, cette langue étant apparue à Molnár comme une langue « difisil é onteûz », notamment "parske lefransè, pour unn orèy ongroiz é tunn lang ki tourn pa ron» (p. 14-15). Par ailleurs, les circonstances de son apprentissage ont occasionné beaucoup de vexations à la poète. À l'université, ses professeurs, presque 
tous des Hongrois qui n'étaient pas allés en France, fondaient leur enseignement sur l'écrit, c'est-à-dire sur l'orthographe, la grammaire et la lecture des classiques. La seule Française de l'établissement leur donnait des dictées pleines de pièges et renforçait le caractère humiliant de l'exercice en exigeant des étudiants qu'ils annoncent à voix haute le nombre de fautes qu'ils avaient faites : «-É koi? Ankor karant fôt! Ankor sinkant fôt! » (p. 16) L'humiliation ne s'arrêta pas là, comme elle le raconte ensuite :

[avant de venir en France] chparlè pa, chparlè peû, chparlè mal, toutfasson, chkonprenè très mal skon me dizè mé kan chparlè, chparlè kom Kornèy é Rassin :

- Ô kruèl souvenir de ma gloire passé! Euvre de tan jour en un jour éfassé!

Parske sété ègzakteman sa : jariv an Frans avèk tou ske jé apri, le vieû fransè él latin é la gramèr é la litératur é ke "amour» dvien féminin ô plurièl é patati é patata épui jariv an Frans é la, stupéfaksion total : mé il parl koi, sé janla? (p. 16)

Ils parlent quoi ? Un français qu'elle ne connaissait pas, qu'elle appellera par la suite le parlé ou le parlé populaire. Elle a mis des années à apprendre ce deuxième français, a beaucoup souffert des moqueries de l'entourage, elle qui s'exprimait trop lentement en essayant de bien suivre les règles de grammaire. Cette expérience la conduit à une réflexion sur le fonctionnement du français et des langues en général, dont la portée est à la fois sociale, philosophique et poétique.

\section{Le respect, baoui ! lerèspè des fautes autorisées}

La découverte du français parlé amène Molnár à faire une sévère critique de l'écrit, seule norme reconnue et objet d'une valorisation qu'elle juge excessive. Elle déplore le 
conservatisme de cette langue écrite, son clivage d'avec la parole quotidienne, clivage manifeste non seulement dans sa graphie, mais aussi dans sa grammaire, pleine de formes qui ne s'emploient plus à l'oral, comme certains temps verbaux et le style indirect. Socialement, cette situation a des conséquences : elle rend difficile l'acquisition de l'écrit, en particulier pour les immigrés. Dans «Du français» et konférans pour lézilétrés, Molnár propose une écriture phonétique plus facile à apprendre que l'autre, qui pourrait coexister avec elle et servir dans le quotidien pour permettre à plus de gens de lire. Sa proposition ne s'arrête pas à la démocratisation de la lecture : elle croit aussi qu'il faut écrire la langue parlée dans sa grammaire, son vocabulaire et sa syntaxe propres. Elle déplore que cette langue soit méprisée par la grammaire et les instances officielles, car elle est ainsi méconnue. Elle la compare à « un fantôme ", parce que non étudiée ${ }^{5}$, "sans règles apparentes » (1997, p. 123). Si le parlé était mieux connu, on pourrait enseigner aux enfants et aux illettrés le fonctionnement de la langue qu'ils parlent: «l'école serait moins assujettie à la névrose correctionnelle» (1997, p. 125). Pour elle-même, Molnár dit aimer la parole parce que les fautes ne peuvent y être vérifiées (1996, texte 25). Enfin, elle juge que le français écrit est une langue désuète, fixée au XVIIe siècle, alors que le parlé est beaucoup plus souple, plus libre, moins soumis aux

\footnotetext{
${ }^{5}$ En fait la langue parlée est analysée par les linguistes : j'imagine qu'elle n'en parle pas parce que ces travaux ne franchissent guère le cercle des spécialistes. Voir par exemple Claire Blanche-Benveniste, Approches de la langue parlée en français (2003), Claire Blanche-Benveniste et Colette Jeanjean, Le Français parlé (1987), Françoise Gadet, La Variation sociale en français (2007) et « De quelques textes fondamentaux sur l'oral », " Construire une problématique de l'oral» (séminaire doctoral de Sciences du langage du 3 juin).
} 
règles et donc plus vivant. On reconnaît dans ses propos le vieux dualisme entre la voix vive et la lettre morte, souvent reconduit pour le cas du français, dont l'écrit, réglé par des institutions puissantes, intègre peu les transformations du parlé. Raymond Queneau, dans les années 1930 déjà, avait formulé des réflexions semblables à celles de Molnár, déclarant «mort » le français des grammaires, plaidant pour l'adoption d'une orthographe phonétique apte à rendre compte de la langue parlée, qu'il appelait le «néo-français ${ }^{6}$ ».

Cependant, le problème de la norme, pour Molnár, ne se limite pas à la différence entre l'écrit et le parlé, ni au cas du français: si elle l'illustre avec cette langue pour des raisons évidentes, il s'étend à tous les idiomes. La poète observe l'absence de relation directe entre les règles de grammaire et une bonne communication, qui est aussi l'absence de rapport entre la norme et ce qui pourrait être une logique interne à la langue. Elle donne l'exemple d'un enfant qui, spontanément, construit le passé composé de «prendre» comme celui de «tendre» ou de «rendre», dit «j'ai prendu», comme on dit «j'ai tendu » ou « rendu ». Cette absence de nécessité des règles se marque aussi par le fait que «le phénomène correct devient une faute grave et inversement, la faute grave devient un phénomène correct en fonction de la langue dans laquelle on se trouve, édela delà onpeudirke, on peut déduire que la correction linguistique n'est rien d'autre [...] que le respect, baoui! lerèspè des fautes autorisées » (1998). Elle décrit les

\footnotetext{
${ }^{6}$ Voir Raymond Queneau, Bâtons, chiffres et lettres (1965), en particulier la section «Préliminaires », qui contient plusieurs textes sur le sujet. Pour son analyse des différences entre les deux langues, il s'inspirait notamment des travaux de Joseph Vendryès (Le langage, 1921) et de Wilhelm von Wartburg (Évolution et structure de la langue française, 1934).
} 
fautes comme résultat de contradictions internes à une langue ( «tendre » qui fait «j'ai tendu» et "prendre» qui fait «j'ai pris »), ou encore de contradictions existant entre deux langues différentes. Elle relie cela encore à des problèmes sociaux, ceux de l'inclusion ou de l'exclusion (la correction linguistique signale l'appartenance à une nation), ceux de l'autorité et de la soumission (ceux qui excellent à parler selon la norme sont les plus soumis).

\section{la langue correcte, ne peut garantir, skimétarivé danlpassé, non}

L'arbitraire de la norme inspire aussi à Molnár des réflexions d'ordre philosophique, existentiel, sur l'inadéquation entre la grammaire et l'expérience du monde: «les phénomènes langagiers ne reflètent pas les phénomènes réels, aça non! $\mathrm{Ne}$ les traduisent pas, ne les expriment pas fidèlement, tuçavè? pour la simple raison qu'il n'y a aucune loi, aucune convention qui régit les constructions langagières » (1998). Elle illustre cela par l'absence de lien entre la relation au passé et la correction dans l'emploi des temps verbaux avec leur valeur aspectuelle : «La langue, pas même la langue correcte, ne peut garantir, skimétarivé danlpassé, non [...]. Si l'on suppose que la justesse langagière est de reproduire, d'une façon plus ou moins fidèle, les phénomènes réels dans la langue, parske cétèribl! la correction linguistique n'y est pour rien.» (1998) Non seulement le choix de telle ou telle forme n'atteste pas de la vérité de ce qui s'est produit, mais les explications fournies par les grammaires ne coïncident pas avec l'expérience et mènent à des déconvenues ceux qui essaient de se baser sur elles pour s'exprimer : «parskemoi, kiçuipafrançèz, jessèydabordéléchôz atraverlérègl, éalorla, achakfoi, jramass démèrd » (1998). 
Cet ensemble de constats d'arbitraire conduit logiquement l'écrivaine à affirmer l'autonomie des phénomènes langagiers. À cette occasion, elle fait quelques réflexions sur le lexique, qui, encore une fois, mettent en relation les normes linguistiques et les rapports sociaux. Elle évoque les associations sémantiques qui n'ont rien à voir avec le monde matériel et donne l'exemple d'un mot, "cochon », qui désigne un être physique, un mammifère, mais qui qualifie aussi, dans une comparaison, une manière malpropre de manger, dont on se sert ensuite pour construire des insultes du type « cochon de Juif ». Pour Molnár, l'autonomie signifie donc, et surtout, que les phénomènes verbaux ne font pas que décrire la réalité (et quand ils le font, il le font de manière imparfaite, partielle et partiale), mais il servent surtout à la manipuler, à exercer une domination sur elle, notamment parce qu'une fabricabilité infinie est inhérente au langage, laquelle n'est réglée par aucun principe de vérité.

\section{écéla justeman ouçafoir ôjourdui avèklalitératur}

Par rapport aux réflexions sur la langue, la part consacrée à la poétique proprement dite occupe peu de place dans les essais de Molnár. Sa vision de la littérature est inséparable de ses idées linguistiques. Le plus long passage de poétique - si l'on excepte la suite «Quant à je », qui est dans l'œuvre - se trouve dans «Dlalang» et commence ainsi : «dlalang litérèr? jépagrandchôz adir ladçu, toutfaçon, jédjatoudi. bon.» Elle n'avait pas exactement tout dit avant d'aborder la littérature, mais presque, puisque la langue littéraire est directement affectée par le fossé qui sépare la correction langagière de l'expérience. Pour Molnár, l'écrivain doit éviter toute forme de 
purisme, refuser de se laisser enfermer par le code et user des possibles de la langue pour créer des textes qui produisent du plaisir. Ce qu'elle étaie par des considérations historiques: «Pendant de longs siècles, les langues nationales correctes n'existaient pas encore ${ }^{7}$ » (1998), écrit-elle. Autrement dit, elles étaient encore peu normées, du fait que la langue d'écriture était surtout le latin. La littérature, comme d'autres types de discours, a servi à légitimer les langues vulgaires, à montrer qu'on pouvait y dire tout, pour finalement contribuer à leur normalisation. Tout ce processus a finalement donné lieu à une association étroite entre la littérature et la langue nationale correcte : " écéla justeman oaçafoir ôjourdui avèklalitératur » (1998). L'écrivain qui s'assujettit à la norme s'interdit de manier le langage librement, contribue à dissimuler l'absence de conformité entre le langage et le monde extra-linguistique, il fait comme si le langage correct était transparent, comme s'il pouvait garantir une vérité, par exemple attester de «skimétarivé danlpassé ». Un tel écrivain "devient émetteur d'idées ou de sentiments » (1998), au lieu d'exercer sa compétence propre, de produire des textes qui, en plus de faire plaisir, ont pour effet de mettre au jour «ce qui dans la manipulation langagière ordinaire est masqué », c'est-à-dire « la fabricabilité à l'infini des objets langagiers » (1998). Révéler l'artifice du langage en explorant son potentiel créatif et jouissif : la fonction de la littérature est à la fois hédoniste et critique.

7 Le parallèle que fait Molnár entre le français parlé et les langues vernaculaires du Moyen Âge, d'une part, et entre le français écrit et le latin, d'autre part, se trouve aussi chez Raymond Queneau. 
On retrouve chez Molnár plusieurs thèmes privilégiés de la réflexion linguistique des poètes, notamment ceux que j'ai évoqués plus haut, soit l'arbitraire des langues et le caractère aliénant potentiel du langage partagé. On pourrait en mentionner d'autres, par exemple celui de la critique de l'illusion réaliste, si souvent réitérée depuis que Mallarmé a décrété «Abolie, la prétention, [...] d'inclure au papier subtil du volume » le bois même des arbres ou les pierres «sur quoi les pages se refermeraient mal » (p. 210) et qui a souvent donné lieu à des poétiques intransitives, centrées sur les «objets langagiers ». Molnár ne prétend d'ailleurs à aucune originalité, ce qui est manifeste dans le texte d'ouverture de Poézi prolétèr que j'ai cité tout à l'heure : «il fô, ilfô reprandr trankilman letruk édir dé, redir déchôz toutsinpl, ki onbezoin de, de réafirmé. »Cela dit, sa poétique a sa spécificité, ne serait-ce que dans l'agencement de ces choses toutes simples qui ont besoin d'être réaffirmées.

Les récriminations des poètes concernant l'arbitraire portent le plus souvent, comme dans le Cratyle, sur l'absence de relation analogique entre les mots (dans leur signifiant phonique ou graphique) et les choses ou entre les mots et le corps. Molnár pense l'arbitraire au plan grammatical, syntaxique et pragmatique du discours, à partir des redondances et contradictions internes aux langues, à partir aussi des différences entre elles. Quand elle aborde le lexique, ce n'est pas pour lui reprocher son manque de ressemblance avec les choses désignées, mais pour montrer comme il est facilement chargé de connotations axiologiques, affectives, etc., ce qui, entre autres, le rend utilisable pour manipuler la réalité8.

\footnotetext{
${ }^{8}$ Voir dans « Dlalang » et dans « Du français », Poézi prolétèr (p. 129-132).
} 
Par ailleurs, contrairement à ce qu'on a pu voir chez de nombreux poètes, la poésie, la littérature ne se voient aucunement chez elle octroyer la fonction de remotiver le langage pour le rapprocher du monde, du corps ou de l'intimité: l'absence de justesse du verbe par rapport à l'expérience ne peut être corrigée, si bien que la " résistance », à ses yeux, consiste à exhiber la distance entre les phénomènes réels et langagiers en usant de tout le potentiel de création dans la langue. Il semble que, dans une telle perspective, tenter une remotivation serait reconduire autrement l'illusion réaliste, faire croire qu'une description du monde peut être juste et neutre.

Les réflexions de Molnár sur la « fabricabilité des objets langagiers » présentent par ailleurs des affinités avec les théories de Noam Chomsky, notamment avec la thèse de la « créativité infinie» du langage : «[0]nce we have mastered a language, the class of sentences with which we can operate fluently and without difficulty or hesitation is so vast that for all practical purposes [...] we may regard it as infinite. » (1964, p. 7) Dans l'entretien qui fut réalisé en 2007 par Libr-Critique (une dizaine d'années après la parution des essais et œuvres que j'analyse ici), Molnár parle abondamment de Chomsky9. Elle y aborde la résistance qu'elle rencontre lorsqu'elle veut discuter des thèses chomskyennes avec les intellectuels français, en particulier à cause des positions du linguiste américain dans l'affaire Faurisson ainsi que de ses thèses sur l'innéisme biologique du langage, l'innéisme étant souvent

\footnotetext{
${ }^{9}$ Molnár y raconte qu'elle a découvert Chomsky très tôt, dans les années 1970, alors qu'elle étudiait la linguistique à l'université. Alors que le régime censurait à peu près tout, la linguistique générale avait échappé à sa vigilance et ses professeurs traduisaient les premières œuvres de Chomsky.
} 
associé au fascisme. Si elle juge négativement les vues sur Faurisson, elle défend l'innéisme, parce qu'il rend possible « l'aspect créateur » du langage : il y a, au fond de toute langue, un système automatique, "un système organique qui nous permet d'exprimer des significations illimitées et des pensées illimitées ${ }^{10} »$. Molnár voit dans le lien entre innéisme et créativité la possibilité d'une liberté pour tous : « tout le monde de la même manière a la faculté de la langue et aura la langue et va pratiquer la langue et il n'y a pas... aucun régime ne peut enlever de l'être humain cette capacité ». La réflexion qu'elle proposait en 1997 dans Dlalang ne traitait pas de cette liberté inhérente à une faculté naturelle de langue: avec l'idée de «fabricabilité », elle mettait davantage l'accent sur l'absence de conformité entre la construction des discours et le monde empirique, les dangers de manipulation qui en résultaient et la nécessité pour l'écrivain de mettre à nu le caractère d'artefact du langage.

\section{dans la langue hongroise, on n'a qu'un œil, qu'une oreille, qu'une fesse...}

La vision de Molnár de l'autonomie linguistique se rapproche aussi des thèses de Chomsky, sauf que, chez celui-ci, la séparation entre langage et réel est encore plus radicale que chez la poète :

[0]ne fundamental contribution of what we have been calling 'Cartesian linguistics' is the observation that human language, in its normal use, is free from the control of independently identifiable external stimuli or internal states and is not

\footnotetext{
${ }^{10}$ Transcrit de l'interview de Libr-Critique.
} 
restricted to any practical communicative function, in contrast, for example, to the pseudo language of animals. (1966, p. 29)

The general conclusion that seems to come to the fore, $[\ldots]$ is that language is designed as a system that is « beautiful » but in general unusable. It is designed for elegance, not for use, though with features that enable it to be used for the purposes of normal life (Chomsky, 1991, p. 49; cité par Trabant, 2003, p. 138).

La langue, selon Chomsky, n'est pas d'abord faite pour la communication, elle est plutôt cognition. Sauf qu'il ne s'agit plus ici de la fonction cognitive qu'on assignait traditionnellement au langage, à savoir de forger des concepts aptes à représenter le monde, comme l'explique Jürgen Trabant : « Le langage [chez Chomsky] n'a absolument aucune relation au monde, ni aux choses (res) ni aux autres hommes. Il est sans monde» (p. 138). Et cela, parce que ce qui intéresse le penseur américain, c'est le dispositif cognitif inné, qui, en fait, n'est plus « langagier », mais pur mécanisme mental, totalement indépendant des sons et des significations, de la parole, donc, mais aussi des langues, dont la diversité n'a aucune importance : «A rational Martian scientist would probably find the variation rather superficial, concluding that there is one human language with minor varieties » (Chomsky, 2000, p. 118 ; cité par Trabant, 2003, p. 281). Molnár reprend cette idée quand elle propose d'élargir l'idée de la correction linguistique à "tout énoncé compréhensible entre deux interlocuteurs ». Un tel élargissement «n'est pas loin de cette constatation de Chomsky : il y a plusieurs langues mais les variations sont très superficielles » (1996a), commente-t-elle.

Mais la séparation que fait le linguiste entre une langue intérieure strictement cognitive et une langue extérieure dont les sons et les significations seraient des réalisations 
accessoires contredit certains aspects de la réflexion et de la poétique de Molnár, notamment le rôle que joue chaque idiome (le hongrois et le français pour elle) avec son découpage conceptuel et sonore propre, dans la sensation du corps et la perception du monde extérieur :

De plus, toujours malgré moi et comme tout le monde, ma langue d'origine conditionne mes sensations. Par exemple, je pense et je dis : "J'ai mal à une dent», au lieu de dire «j'ai mal aux dents », ce qui est fautif en français. Mais dans la langue hongroise, on n'a qu'un œil, qu'une oreille, qu'une fesse, qu'une jambe, qu'un bras, et lorsqu'on est manchot, on n'a plus qu'un demi-bras. La perception intime de l'anatomie est donc différente pour un Hongrois et pour un Français. Les deux langues que je pratique s'influencent, se télescopent, entrent en conflit (cité par Diatkine, 1996, p. 4).

Si Molnár constate un écart irrécusable entre la réalité et le langage infiniment "fabricable», écart que l'écrivain ne cherchera pas à annuler par quelque remotivation, mais à rendre le plus manifeste possible, sa conception de l'autonomie linguistique ne saurait être assimilée à celle de Chomsky, puisqu'elle est issue des divergences de visions du monde associées aux différentes langues. Et même, bien que la poète s'exprime encore ici en évoquant la norme (" ce qui est fautif»), le phénomène dont elle parle ne relève pas de la simple correction linguistique, mais de la manière dont le langage s'entrelace à toute notre expérience. Par ailleurs, la perception qu'a l'écrivaine des deux français, l'écrit savant, figé, académique, et le parlé vivant, libre, créatif, ne s'accorde guère elle non plus avec l'idée selon laquelle les particularités phonétiques, morpho-syntaxiques et sémantiques d'une langue ne seraient que des variations de peu d'importance, des «normes » sans effet sur notre relation au réel, comme si les 
unités linguistiques n'étaient que des instruments. Et il en est de même de tout le travail opéré dans son œuvre sur le signifiant, par exemple les transpositions du parlé, l'usage d'une orthographe et d'une syntaxe archaïsantes, la contamination du français par la phonétique, la morphologie et la grammaire hongroises.

\section{le bèrjé soufle son chagrèn dan sa kornemuz é le lès partir avèk le van}

Car une théorie du langage et une poétique ne se révèlent pas que dans des explications, mais également dans ce que fait le discours. Or, il y a un aspect central de cette poétique en acte dont les essais ne parlent pas : le rôle de la transposition de la langue parlée dans la poésie. Alors que Raymond Queneau réclame une « ortograf fonétik » $(1965$, p. 26$)$ et une écriture du « néo-français » pour des motifs littéraires, l'écrivaine francohongroise insiste beaucoup sur les raisons pratiques, sociales et politiques d'écrire le français parlé. Queneau voyait la nouvelle langue comme la condition d'une nouvelle poésie et croyait que l'accomplissement de celle-ci exigeait l'écriture de celle-là : «sans une notation correcte du français parlé, il sera impossible (il sera himpossible) au poète de prendre conscience de rythmes authentiques, de sonorités exactes, de la véritable musique du langage. Car c'est de là que sourd la poésie. » (p. 21) Outre une ou deux allusions dans des notes, Molnár, à ma connaissance, ne s'explique réellement sur l'intérêt poétique du français parlé que dans l'entretien de Librcritique, grâce à une question de l'interviewer. Pourtant, dans la facture de ses écrits, poèmes, roman et entours, la transposition du parlé est omniprésente. 
On trouve un état embryonnaire de cette transcription dans les notes de son premier livre, poèmesIncorrects et mawvaisChants chantsTranscrits. Dans sa préface à ce livre, Molnár distingue deux types de notes ${ }^{11}$ : les "personnelles», qui usent d'une écriture phonétique, et les autres, dont l'orthographe est standard, mais qui sont en italique (p. 7). Les notes personnelles sont dominantes: elles commentent non seulement les poèmes et les chants, mais aussi le titre, le dispositif du livre, la graphie du nom de l'auteure, etc. Ces explications sont en partie techniques (portant sur les modes d'écriture et les sources des textes), en partie autobiographiques. La partie du titre "poèmesIncorrects » est justifiée en note par le rapport hésitant de l'auteure à ses langues (hongrois et français) et l'enracinement de cette difficulté dans son histoire familiale. Molnár décrit ensuite, dans la même glose, l'origine et le procédé d'écriture des poèmes :

Mon frèr éné m'a unn foi anvoayé unn lètr kodé. Il i ranplasè chak mô par lé kordoné de sa plas dan le diksyonèr (le numéro $\mathrm{d}$ paj é le numérô d lign). Il m'a falu unn aprè-midi antyèr pour déchifré sa ltr é sa m’a égzaspéré. Mè le rézulta a été surprenan : oen méssaj prèske san gramèr. Oen peu konfu mé konpréansibl. Sa m'a tou d suit doné anvi d'ékrir de sèt sort : mé poèmesIncorrects son né ènsi. D’abor dan ma lang maternèl, ansuit an fransè ${ }^{12}$. (p. 2, note 1 )

Le reste du titre, en particulier le segment mawvaisChants avec sa biffure, reçoit aussi une longue explication en note. Le « mauvais » devait indiquer à la fois le caractère inchantable des textes après la transcription (laquelle obéit au principe des

\footnotetext{
11 Comme je l'ai mentionné au début de cet article, les notes composent presque la moitié de ce livre.

12 Cela est la suite de la citation présentée au début de la section « Ô kruel souvenir de ma gloire pasé! »
} 
poèmesIncorrects) et un jugement de valeur de l'auteur sur leur qualité, jugement qu'elle a décidé de raturer après avoir constaté que quelques-uns étaient bons, comme le chantTranscritAvecBerger, dont on trouve deux versions, l'une en bonne page, l'autre dans la page des notes et autres entours :

\author{
chantTranscritAvecBerger \\ bergerÊtreHeureux \\ carPousserSonTroupeau \\ depuisCollineJusqueColline \\ etSoufflerDansSaFlûte \\ etSansChagrinVivreSaVie \\ etSiSaFlûteL'ennuyer \\ sortirAlorsSaCornemuse \\ etSoufflerDedansSonChagrin \\ etLaisserLePartirAvecVent (p. 54)
}

Il y a également un arrière-plan biographique aux "mauvaisChants »: Molnár raconte qu'elle chantait beaucoup en Hongrie, avec son père, à l'école, dans des groupes de jeunes, etc. Si bien que ces chansons populaires - enfantines, patriotiques, fascistes, militaires, tziganes, révolutionnaires, etc. -, qu'elles soient bonnes ou mauvaises, ont traversé, d'une façon ou d'une autre, son quotidien de petite fille et d'adolescente : elles font partie de son histoire.

Le mode de présentation du livre, avec poèmes et chants à droite et notes ou documents à gauche, fait également l'objet d'une note, dont l'appel suit le nom de la collection. En Hongrie communiste, raconte Molnár, tous les écoliers et étudiants devaient porter un uniforme, un blouson; elle avait essayé de marquer sa différence en portant une jupe très courte, mais sa note de comportement avait baissé, elle avait donc dû se conformer. Depuis, ce goût adolescent de se distinguer lui était passé... mais pas tant que ça, puisque les collections des 
éditeurs lui font penser aux blousons et qu'elle voulait signaler ce qu'était pour elle un livre, " un objet personnel». Un autre aspect très singulier du livre, la transcription phonétique des notes, appelle par ailleurs un commentaire fort révélateur : « [la transcription phonétique] m'a servi d'ènstruman tèl la kornemuz du berjé dan la chanson : "le bèrjé soufle son chagrèn dan sa kornemuz é le lès partir avèk le van”. »

Parmi les notes, celles que Molnár qualifie de «personnelles» le sont donc de deux manières: par la transcription phonétique et la dimension autobiographique. Or, ce double caractère subjectif vient donner un tout autre éclairage à la théorie linguistique et poétique de l'auteur. L'écriture phonétique, ne reprenant du parlé à peu près que des traits de prononciation, est néanmoins une première version de la transcription du français populaire qui sera si importante dans la réflexion théorique et la suite de l'œuvre. Or, cette transposition est dans ce livre clairement associée à un instrument personnel, aux souvenirs, aux affects - en particulier au chagrin, par la comparaison avec la cornemuse du berger. Dans les notes de Quant à je, Molnár appelle «ékri dlavoi » ces transcriptions, soulignant par là leur matérialité et leur lien avec le corps. Tout cela ramène, de manière indirecte, l'ancienne association entre la poésie, la voix et l'émotion. Dans l'entrevue donnée à Libr-critique douze ans plus tard, Molnár confirme ce rapprochement en disant que la langue parlée, proche de l'émotion, est un matériau poétique par excellence. Quant aux parties autobiographiques des notes, elles viennent, dans une sorte de contrepoint, doubler, amplifier, compléter les chants et poèmes en narrant des circonstances ou des anecdotes vécues qui les sous-tendent ou leur donnent sens par association. 
Le «je » autobiographique et le «je » des poèmes s'identifient alors l'un à l'autre, dans une variante particulière de l'association traditionnelle entre la figure du sujet lyrique et celle de l'auteur. Au fond, les notes personnelles viennent ici réintroduire la voix et l'anecdote qui se trouvent entravées ou brouillées dans la facture particulière des poèmesIncorrects, presque sans grammaire, et les chantsTranscrits, devenus inchantables. Écriture phonétique et textes sans grammaire sont aussi, comme elle le dit explicitement, une étape dans la quête d'une langue plus permissive: un coup de pied à la norme, une manière d'échapper à l'humiliation des fautes, de venger les hontes anciennes ou récentes en pratiquant un langage impur.

Mais il y a plus. Dans le contrepoint que tressent les notes, les poèmesIncorrects et les chantsTranscrits, se crée une tension intéressante entre, d'une part, des caractéristiques du discours, le parlé et le témoignage, associées à une recherche d'authenticité ${ }^{13}$, à une grande proximité entre le langage et la vie et, d'autre part, des éléments qui, au contraire, affichent l'artifice, la distance face à la référence, par la défiguration de la grammaire, la citation et la défiguration des citations. Le souci de marquer le texte d'une empreinte personnelle, d'y inscrire la voix, les émotions, la mémoire, la biographie - qu'on trouve non seulement dans ce premier livre, mais, sous d'autres formes, dans tous les autres - paraît contradictoire avec la théorie poétique de Molnár, qui dit que les écrivains ne doivent pas masquer le manque d'adéquation entre les phénomènes réels et les énoncés, mais montrer la fabricabilité de ces derniers. Sauf que, si on y réfléchit, c'est précisément l'un des

\footnotetext{
${ }^{13}$ Culturellement et dans les propos de Molnár elle-même.
} 
effets du contrepoint entre une parole d'allure «naturelle » et l'autre, ostensiblement "construite», que d'exhiber l'artefact qu'est le langage. Même à l'intérieur des notes, la tension entre authenticité et fabrication apparaît, parce que la transcription phonétique, loin de nos habitudes de lecture, commence par briser l'illusion de transparence du langage avant d'induire la sensation de proximité d'une voix. La poétique des «objets langagiers » de Molnár n'est donc pas un refus de la référence : dans la tradition de la défamiliarisation des formalistes russes ${ }^{14}$ et plus encore de sa reformulation brechtienne, la distanciation, la poète opère une " mise à nu du procédé » et celle-ci fait voir la réalité d'une manière nouvelle. Ce qui est évidemment antinomique avec la conception chomskyenne de l'autonomie linguistique. Chez Molnar, ce qui est senti, saisi, appréhendé autrement, c'est donc à la fois la référence et le langage. Ensemble, l'usage de la transcription et celui du contrepoint induisent de l'hétérogénéité, une division, une multiplicité internes au discours. Ce déchirement, cette distance sont marquées jusque dans le prénom de l'auteur, double, comme l'indique la graphie de la page titre : $\operatorname{Kat}(\mathrm{al}) \mathrm{i}(\mathrm{n})$, et la note qui en décrit les deux versants :

Le prénon féminèn ongroa Katalin, ofisyèl é kelke peu solanèl n’è pa zutilizé dan la lang kourant ki lui préfèr le diminutif Kati. An revanch, selui-si ne figur jamè sur oen dokuman ofisyèl ou ènprimé. Ènsi, deu variant konplémantèr koégzist dan $s$ prénon : le Katalin ôstèr é oen peu réfrijéran mè mélodyeu (dan

\footnotetext{
${ }^{14}$ Je fais ici référence à la notion d'ostranenie proposée par Viktor Chklovski dans «L'art comme procédé », notion que l'on traduit de diverses façons, notamment «défamiliarisation », «désautomatisation » ou «singularisation »: «Le but de l'art, c'est de donner une sensation de l'objet comme vision et non pas comme reconnaissance ; le procédé de l'art est le procédé de singularisation des objets et le procédé qui consiste à obscurcir la forme, à augment la difficulté et la durée de la perception. » (1965, p. 82)
} 
lekel "in" se pronons "inn") é le très zinodor mè byèn plu chaloeureu Kati.

Dans les textes ultérieurs, la transposition du parlé se raffine et s'intensifie pour se répandre dans le lexique, la syntaxe et l'énonciation. L'interlocution, en particulier, y est de plus en plus marquée, par des adresses, des formes dialoguées, l'exploitation des répétitions (hésitations, recherche de mots, insistance rhétorique, etc.), des ruptures de construction, des éléments phatiques (phatèmes, ponctuants, captateurs, signaux d'écoute), des actes de parole expressifs et conatifs :

s'écarter des phénomènes autorisés de telle ou telle langue, ékout ! ékoutmoi bien ! Utiliser correctement une langue, c'est non! tutromp.

autrement dit, plus on est capable

non ! céarchifô.

plus on devient excellent

non! ça veupadirça.

skiçignifi

non ! cépavrèé

méci ! çaçignifiçaôssi

non ! chuipadakor.

kelépluzèksélan sonlépluçoumi émerdétok !15 (1996a)

Par ailleurs, les variétés de contrepoint se multiplient, en particulier dans "Dlalang», où sont tressés l'écrit et le parlé ainsi que des textes différents, par exemple un propos théorique et un discours parasite qui implique l'interlocution conversation banale, texte de carte postale, ou, comme ici, directions routières et commentaire métalinguistique :

Vous êtes porte Maillot. L'écrivain qui échange sa compétence d'écrivain contre la compétence d'un puriste ou porte de Champerret. S'interdit de manier les phénomènes langagiers librement. [...] Du coup, son travail n'est plus langagier mais

\footnotetext{
${ }^{15}$ Les caractères plus gros indiquent un accent d'intensité. Ils sont de Molnár.
} 
autre chose. Continuez tout droit sur trois kilomètres, vous arrivez sur un pont qui surplombe la Seine et que vous traversez. Il devient émetteur d'idées ou de sentiments, etc. éla, safoir anouvô. Suivez la route sur votre gauche car l'écrivain dont la compétence permet plus qu'à quiconque de prouver à tel quel <kati, célunntéfôt favorit> point la langue est manipulable, à tel quel <kati, célunntéfôt favorit> point il n'y a pas d'équivalence entre les phénomènes réels et les énoncés langagiers, là, vous êtes rue de Paris, cet écrivain n'arrête pas d'essayer de démontrer le contraire, deux cents mètres plus loin prenez à gauche au feu, à savoir que les énoncés langagiers peuvent reproduire fidèlement les phénomènes réels.

Dans cette polyphonie, cette hétérogénéité, où sont explorés les scories, les inachèvements, les affects et les stéréotypies des discours, Molnár ne met pas seulement à nu la «fabricabilité » du langage, son autonomie face au monde physique et son pouvoir de nous le faire apparaître de multiples façons. Elle met en évidence l'hiatus qui sépare le sujet du langage partagé, la difficulté à parler, à se faire entendre, les défaillances et les luttes inhérentes à l'échange, les malentendus, les discordes ou, tout simplement, le peu d'idées articulées dans les propos tenus. Elle cerne bien cet enjeu particulier de son travail poétique dans son entretien, quand elle explique enfin l'intérêt du français parlé :

plus nous sommes émotifs plus nous aurons des difficultés à exprimer à dire des choses, on va répéter on va ça va s'arrêter c'est quelque chose directement poétique si j'entends par la poésie qu'il faut que ça nous touche émotionnellement (Diatkine, 1996, p. 4)

Toute une dimension du discours - physique, corporelle, affective, pragmatique - se trouve ici reconnue comme matière à poésie et explorée dans ce sens, à des lieues aussi 
bien de l'élégance cognitive chomskyenne que de quelque avatar du cratylisme.

\section{Bibliographie}

Blanche-Benveniste, Claire (2003). Approches de la langue parlée en français, Paris, Ophrys.

- et Colette JEAnjean (1987). Le Français parlé, Paris, Didier Érudition.

ChKLovski, Victor (1965). "L'art comme procédé », dans Théorie de la littérature. Textes des Formalistes russes, réunis, présentés et traduits par Tzvetan Todorov, Seuil, p. 74-97.

Chomsky, Noam (1964). Current Issues in Linguistic Theory, The Hague, Mouton.

- (1966). Cartesian Linguistics. A Chapter in the History of Rationalist Thought, New York, Harper and Row.

- (1991). "Linguistics and Cognitive Science: Problems and Mysteries », in Asa Kasher (ed.), The Chomskyan Turn, Cambridge/Oxford, Blackwell.

- (2000). New Horizons in the Study of Language and Mind, Cambridge, Cambridge University Press.

DiATKine, Anne (1996). «ParléVou Molnar? Un ouvrage d'une totale incorrection, par une Hongroise qui a inventé son français. Cevrékoi merdalor ! », Libération, jeudi 11 juillet, p. 4. 
GADET, Françoise (2007). La Variation sociale en français, Paris, Ophrys.

—, «De quelques textes fondamentaux sur l'oral», Construire une problématique de l'oral, Séminaire doctoral de Sciences du langage du 3 juin, Université de Paris X - Nanterre, <http://www.uparis10.fr/1132737585596/0/fiche document/\&RH=recedcc $>$

LAROCHE, Hugues (2007). «Poésie de la linguistique: la tentation du dictionnaire », Semen, Linguistique et poésie : le poème et ses réseaux, no 24, $<$ http://semen.revues.org/document5933.html>

MALlARMÉ, Stéphane (2003). "Crise de vers », dans OEuvres complètes, t. 2, Paris, Gallimard, coll. «Bibliothèque de la Pléiade », p. 208.

MoLNÁR, Katalin (1999). Konférans pour lé zilétrés, Romainville, Al Dante.

— (1997). «Diskusion », Poézi Prolétèr, no 1.

- (1996a). «Dlalang », Revue de littérature générale. 2 Digest, texte 25 .

— (1996b). Quant à je (kantaje), Paris, P.O.L.

MolnÁR, Kat(al)i(n) (1995). poèmesIncorrects et mauvaisChants chantsTranscrits, Nîmes, Fourbis.

Ponge, Francis (1999). «Des raisons d'écrire », Proêmes, dans OEuvres complètes, t. I, Paris, Gallimard, «Bibliothèque de la Pléiade ».

Prigent, Christian (1993-1994). "Chère disparue », Action poétique, «La forme-poésie va-t-elle, peut-elle, doit-elle disparaître? », no133-134, hiver, p. 32. 
QuEneAU, Raymond (1965). Bâtons, chiffres et lettres, Paris, Gallimard.

Roubaud, Jacques (1995). Poésie, etcetera : ménage, Paris, Stock.

TRABANT Jürgen (2003). Mithridates im Paradies. Kleine Geschichte des Sprachdenkens, München, C.H.Beck.

\title{
Résumé
}

Le présent article aborde le rapport entre théorie du langage et poétique dans les " entours » - articles, manifestes, entrevues, préfaces et notes - de l'œuvre de Katalin Molnár, poète française d'origine hongroise. Depuis Mallarmé, nombreux sont les poètes français qui ont déploré les "défauts des langues", assignant à la poésie la tâche de les pallier. Si Molnár reprend certaines doléances de ses prédécesseurs, par exemple sur l'arbitraire linguistique et sur le rapport entre communication et pouvoir, sa théorie se démarque à maints égards des leurs. Et les solutions poétiques qu'elle propose manifestent un déplacement par rapport à la tradition : elle cherche moins à remédier aux défauts de la langue qu'à les exhiber, à jouer avec les manques, les stéréotypies, les pauvretés de la parole.

\begin{abstract}
This article approaches the Relationship between language theory and poetics in the margins (articles, manifestos, interviews, prefaces and footnotes) of Katalin Molnár's work. Since the end of 19 th century, many poets, following Mallarmé, have complained about imperfections of languages, and assigned to poetry the aim of compensating for their defaults. Molnár, a French writer born and educated in Hungary, take up
\end{abstract}


some of her predecessors' complaints: she laments, for instance, the arbitrary character of language and the relationship between communication and power. But her theory shows also many differences from theirs. And the poetic "solutions" she puts forward bring something new in this tradition: she doesn't try so much to remedy language imperfections as to expose them and to play with the deficiencies, the stereotypes, the poverties of speech. 